\title{
Avaliação do desempenho de parâmetros imunológicos como indicadores de progressão clínica da infecção crônica pelo HTLV-1
}

\author{
Evaluation of the performance of immunological parameters as indicators for \\ clinical progression of chronic HTLV-1 infection
}

\author{
Jordana Grazziela Alves Coelho-dos-Reis ${ }^{1,2}$, Roberta Dias Rodrigues Rocha ${ }^{1}$, \\ Gustavo Eustáquio Alvim Brito-Melo ${ }^{3}$, João Gabriel Ribas ${ }^{4}$, Anna Bárbara de Freitas \\ Carneiro-Proietti ${ }^{5}$, Bernadete Catalan-Soares ${ }^{5}$, Edel Figueiredo Barbosa-Stancioli ${ }^{2}$, \\ Grupo Interdisciplinar de Pesquisas em HTLV $^{5}$ e Olindo Assis Martins-Filho ${ }^{1}$
}

\begin{abstract}
RESUMO
Neste estudo, foi avaliado o desempenho isolado e combinado de parâmetros laboratoriais, percentual de linfócitos $B$ (\%LB), a razão entre células $T / B$ e o \%CD8 ${ }^{+} H L A-D R^{+} / C D 8^{+}$, na identificação de indivíduos assintomáticos-AS ou portadores de HAM/TSP-HT numa população de casos soropositivos para HTLV-1. Índices expressos em porcentagem demonstram que cada parâmetro, isoladamente, apresenta desempenho moderado, com co-negatividade $=83 \%$ e $91 \%$ para \% LB e razão entre células $T / B$, respectivamente e co-positividade $=78 \%$ para $\% C D 8^{+} H L A-D R^{+} / C D 8^{+}$. A análise combinada $\left(\% C D 8^{+} H L A-D R^{+} / C D 8^{+}\right.$e razão $T / B)$ não revelou ganho significativo no desempenho (co-positividade $=75 \%$, co-negatividade $=74 \%$ ). A análise das razões de verossimilhança em diferentes faixas de valores, para os parâmetros isolados, revelou que um indivíduo soropositivo para HTLV-1 com $\% L B<7 \%$, razão entre células $T / B>11$ e $\% C D 8^{+} H L A-D R^{+} / C D 8^{+}>70 \%$ possui, respectivamente, 11, 19 e quase 10 vezes mais chances de pertencer ao grupo HT. Portanto, recomenda-se o uso desses indicadores fenótipos na propedêutica laboratorial complementar de monitoração da progressão clínica da infecção crônica pelo HTLV-1.
\end{abstract}

Palavras-chaves: HTLV-1. Imunofenotipagem. Índices de desempenho. Indicadores de morbidade.

\begin{abstract}
This study evaluated the performance of single and combined laboratory parameters, B-lymphocyte percentages (\%LB), T/B cell ratio and $\% C D 8^{+} H L A-D R^{+} / C D 8^{+}$, to differentiate asymptomatic cases (AS) from HAM/TSP patients (HT) within a population of HTLV-1 seropositive cases. Percentage indices demonstrated that each parameter alone presented moderate performance, with co-negativity of 83 and $91 \%$ for $\% \mathrm{LB}$ and T/B cell ratio, respectively, and co-positivity of $78 \%$ for $\% C D 8^{+} H L A-D R^{+} / C D 8^{+}$. Combined analysis (\%CD8 ${ }^{+} H L A-D R^{+} / C D 8^{+}$and T/B cell ratio) did not show any substantial performance enhancement (co-positivity $=75 \%$ and co-negativity $=74 \%)$. Likelihood ratio analysis using different value ranges for the separate parameters revealed that $H T L V$ 1 seropositive cases with $\% L B<7 \%$, T/B cell ratio $>11$ and $\% C D 8+H L A-D R+/ C D 8+>70 \%$ would have, respectively, 11,19 and 10 times greater chance of belonging to the HT group. Therefore, use of these phenotypic indicators as complementary laboratory methods for monitoring the clinical progression of chronic HTLV-1 infection is recommended.
\end{abstract}

Key-words: HTLV-1. Immunophenotyping. Performance indices. Morbidity indicators.

\footnotetext{
1. Centro de Pesquisas René Rachou, Fundação Oswaldo Cruz, Belo Horizonte, MG. 2. Departamento de Microbiologia do Instituto de Ciências Biológicas, Universidade Federal de Minas Gerais, Belo Horizonte, MG. 3. Universidade Federal dos Vales do Jequitinhonha e Mucuri, Diamantina, MG. 4.Hospital Sarah Kubitschek, Belo Horizonte, MG. 5. Fundação Centro de Hematologia e Hemoterapia de Minas Gerais, HEMOMINAS, Belo Horizonte, MG.

Apoio Financeiro: PIBIC/CNPq, CPqRR/FIOCRUZ e Fundação HEMOMINAS.

Endereço para correspondência: Dr. Olindo Assis Martins-Filho. Laboratório de Doença de Chagas/CPqRR/FIOCRUZ. Av. Augusto de Lima 1715, Barro Preto, 30190-002 Belo Horizonte, MG, Brasil.

Tel: $55313349-7764$

e-mail: oamfilho@cpqrr.fiocruz.br, jordana@cpqrr.fiocruz.br

Recebido para publicação em 27/4/2006

Aceito em 12/1/2007
} 
0 vírus linfotrópico de células T humanas (HTLV) foi 0 primeiro retrovírus descrito em humanos ${ }^{15}$. Estimativas apontam para 15 a 20 milhões de pessoas infectadas pelo HTLV-1 em todo 0 mund $0^{17}$. Estima-se que cerca de 2,5 milhões de pessoas estão infectadas pelo HTLV-1 no Brasil ${ }^{34}$.

O HTLV é um vírus da família Retroviridae classificado em tipos 1 e 2 com tropismo preferencial para linfócitos $\mathrm{T} \mathrm{CD}^{+}$, podendo, entretanto ser detectado em outras populações linfocitárias ou em células da linhagem monocítica/macrofágica ${ }^{10}{ }^{12}$.

A transmissão do HTLV acontece por meio do contato com linfócitos infectados durante o contato sexual, no trabalho de parto, aleitamento materno, transfusões sanguíneas e compartilhamento de agulhas e seringas contaminadas ${ }^{913}$. Os testes de triagem para HTLV-1/2 foram introduzidos em bancos de sangue de vários países, sendo que no Brasil esta triagem é obrigatória, como estabelecido na portaria 1376/1993 do Ministério da Saúde, tendo em vista o risco de transmissão parenteral, ratificada pela portaria 153/2004.

Do ponto de vista clínico, a maioria (90-95\%) dos indivíduos infectados pelo HTLV-1 pode permanecer assintomática por períodos de tempo longos e variáveis. Esses indivíduos não apresentam nenhum sintoma ou sinal clínico da infecção, com todos os exames clínicos normais. Cerca de $2 \%$ dos indivíduos infectados podem apresentar manifestações neurológicas decorrentes da infecção pelo HTLV-1, levando ao surgimento de alterações motoras e sensitivas ${ }^{14}$. Essa patologia é uma doença neurológica de progressão lenta denominada mielopatia associada ao HTLV/paraparesia espástica tropical - HAM/TSP, que acomete, na maior parte das vezes, indivíduos na faixa etária entre 35 e 49 anos, havendo um predomínio do sexo feminino sobre o masculino $0^{14}$. 0 processo patológico envolve desmielinização perivascular e degeneração axonal, acompanhado de uma resposta inflamatória na região afetada e por um infiltrado de células mononucleares, destruição de fibras nervosas no foco inflamatório levando à perda da capacidade motora-sensorial ${ }^{114}$. No pólo de natureza neoplásica, a leucemia/linfoma de células T do adulto - ATLL associada à infecção pelo HTLV-1 é documentada em cerca de 1 a cada 1.000-2.000 indivíduos soropositivos ${ }^{16}$.

Embora os mecanismos imunopatogênicos específicos da HAM/TSP aindanão estejam totalmente esclarecidos, diversos estudostêm demonstrado a participação do sistemaimunológico no desenvolvimento ou manutenção desta desordem neurológica ${ }^{12711}{ }^{19}$. Na tentativa de estabelecer indicadores laboratoriais da progressão clínica da infecção crônica pelo HTLV-1 para HAM/TSP, algumas investigações relatam a aplicabilidade de parâmetros imunológicos de segregar pacientes portadores de diferentes formas clínicas da infecçã $0^{12}$. Nesse contexto, Brito-Melo e cols ${ }^{1}$ descreveram alterações significativas nos percentuais de linfócitos B (\%LB), da razão entre células T/B e o percentual células $\mathrm{T}^{\mathrm{CD}} 8^{+}$ativadas denominado $\% \mathrm{CD}^{+} \mathrm{HLA}$ $\mathrm{DR}^{+} / \mathrm{CD}^{+}$no sangue periférico de indivíduos infectados pelo HTLV-1, portadores de HAM/TSP (HT), capazes de discriminálos dos demais indivíduos soropositivos para o HTLV-1. Esses dados sugerem que tais alterações imunológicas poderiam ser utilizadas como indicadores laboratoriais de progressão clínica para HAM/TSP.
Diante dessas evidências, nesse estudo, propusemos avaliar o desempenho dos parâmetros fenotípicos sugeridos como indicadores imunológicos laboratoriais da progressão clínica da infecção pelo HTLV-1 para HAM/TSP, empregando diferentes índices de desempenho expressos em percentual e em chance. 0 s resultados desse estudo sugerem o uso desses indicadores fenótipos na propedêutica laboratorial complementar de monitoração da infecção crônica pelo HTLV-1.

\section{MATERIAL E MÉTODOS}

População estudada. Para o estudo fenotípico dos leucócitos do sangue periférico, amostras de sangue, coletadas em EDTA foram obtidas de indivíduos infectados pelo HTLV-1, contatados durante a avaliação clínico-laboratorial na Fundação HEMOMINAS e no Hospital Sarah Kubitschek de Minas Gerais/ Associação das Pioneiras Sociais.

Todos os indivíduos provenientes da Fundação HEMOMINAS, incluídos nesse estudo, apresentavam resultados negativos na sorologia para hepatite B e C, HIV, sífilis e doença de Chagas.

Os indivíduos com sorologia positiva para 0 HTLV-1/2 no teste imunoenzimático (ELISA) foram reavaliados através da pesquisa de anticorpos pelo Western Blotting (WB), sendo posteriormente classificados em negativos, positivos ou indeterminados, de acordo com o perfil de bandas observado. Apenas os indivíduos com resultado positivo no teste de ELISA e no WB foram incluídos nesse estudo. A avaliação clínica especializada desses indivíduos foi realizada pela Dra. Bernadette Catalan Soares, no Serviço de Pesquisa da Fundação HEMOMINAS. Os indivíduos considerados infectados que não apresentam sintomas de HAM/TSP foram classificados como assintomáticos (AS). 0 grupo AS foi constituído por 53 indivíduos assintomáticos com média de idade de 38,5 anos, sendo 23 homens e 30 mulheres.

Todos os pacientes provenientes do Hospital Sarah Kubitschek apresentavam sorologia positiva para o HTLV-1 tanto no teste de ELISA quanto WB. Esses pacientes foram submetidos à avaliação clínica especializada pelo Dr. João Gabriel Ribas e classificados como portadores de sintomas e sinais clínicos característicos de HAM/TSP sendo categorizados no grupo HT. Esse grupo foi constituído por 55 indivíduos, com média de idade de 52 anos, sendo 18 homens e 37 mulheres.

Todos os indivíduos incluídos neste estudo assinaram termo de consentimento aprovado pelos comitês de ética da Fundação HEMOMINAS e do Centro de Pesquisa René Rachou - FIOCRUZ/ BH.

Análise fenotípica de leucócitos do sangue periférico por citometria de fluxo. A análise da série branca foi feita seguindo o método de imunofluorescência recomendado pela Becton Dickinson (USA), modificado como a seguir: $100 \mu \mathrm{L}$ de sangue coletado em EDTA, foram incubados por 20-30 minutos, no escuro, à temperatura ambiente, na presença de $5 \mu \mathrm{L}$ de coquetel de anticorpos monoclonais (mAbs), marcados com fluorocromos distintos (ficoeritrina-PE ou isotiocianato de fluoresceína-FITC), específicos para moléculas de superfície 
celular de linfócitos humanos, empregando as combinações anti-CD3-FITC/anti-CD19-PE e anti-CD8-FITC/anti-HLA-DR-PE. Após a incubação, os eritrócitos foram lisados usando-se $100 \mu \mathrm{L}$ de solução de lise (Optilyse-B, Immunotec-USA) por 5 minutos, seguido da adição de $900 \mu 1$ de água destilada e reincubação por 10 minutos, no escuro, à temperatura ambiente. Após a incubação, os leucócitos foram lavados duas vezes com $1 \mathrm{~mL}$ de tampão (PBS) $0,015 \mathrm{M}$ contendo $0,01 \%$ de azida sódica. A suspensão celular foi então fixada com $500 \mu \mathrm{L}$ de FACS fix solution $(10 \mathrm{~g} / \mathrm{L}$ paraformaldeído, $1 \%$ de cacodilato sódico, $6,65 \mathrm{~g} / \mathrm{L}$ cloreto de sódio e $0,01 \%$ de azida sódica).

A aquisição dos dados imunofenotípicos foi realizada no citômetro de fluxo - FACScalibur (Becton-Dickinson, USA), utilizando o programa Cell-Quest. Todas as etapas da imunofenotipagem celular foram realizadas no Serviço de Hematologia da Fundação HEMOMINAS, sob supervisão do Dr. Olindo Assis Martins Filho e apoio técnico de Saulo Tonholo.

A análise dos dados consistiu da identificação da população celular de interesse (linfócitos), em gráficos de distribuição puntual de caracteres morfométricos de tamanho versus granulosidade (FSC versus SSC) seguida da quantificação percentual das subpopulações celulares (linfócitos T - $\mathrm{CD}_{3}{ }^{+} \mathrm{CD} 19$-, linfócitos $\mathrm{B}-\mathrm{CD}^{-} \mathrm{CD}^{-} 9^{+}$e células T $\mathrm{CD}^{+}$ativadas dentro da

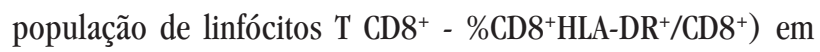
gráficos bidimensionais de intensidade de fluorescência (FL1FITC versus FL2-PE), utilizando o programa Cell-Quest.

Os resultados foram expressos sob a forma de percentual de células positivas para os linfócitos $\mathrm{CD} 3 \mathrm{CD}^{-} 9^{+}$, denominados \%LB. Para obtenção da razão entre células T/B, foi calculado quociente dos valores percentuais de linfócitos $\mathrm{CD}^{+}{ }^{+} \mathrm{CD} 19-$ (LT) e \%LB. 0 valor \%CD $8^{+}$HLA-DR ${ }^{+} / \mathrm{CD}^{+}$foi calculado como a fração de linfócitos T CD8 ${ }^{+}$que expressam o marcador de ativação HLADR, dentre os linfócitos T $\mathrm{CD}^{+}$, através da fórmula: \%CD8 ${ }^{+} \mathrm{HLA}-$

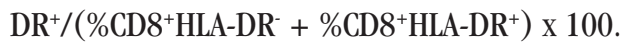

Análise estatística. Os resultados dos valores percentuais das subpopulações de linfócitos analisadas foram submetidos à análise no programa Minitab (Release 13.20) com o intuito de verificar três hipóteses: independência, normalidade e variância dos valores. Uma vez verificado que os grupos de resultados AS e HT não obedeciam a, pelo menos, uma dessas hipóteses, os dados foram considerados como de distribuição não paramétrica. 0 teste de Mann Whitney é 0 indicado para o tratamento estatístico de amostras com distribuição não-paramétrica. 0 teste T de Student é o indicado para o tratamento estatístico de amostras com distribuição paramétrica. As diferenças foram consideradas significativas quando $p<0,05$. Esse tipo de abordagem foi utilizado como critério inicial de avaliação da aplicabilidade dos parâmetros propostos, na identificação dos grupos clínicos avaliados. As análises foram feitas utilizando-se o programa Minitab 3.0.

Avaliação do desempenho dos parâmetros fenotípicos celulares. 0 desempenho dos parâmetros fenotípicos celulares na segregação dos grupos clínicos avaliados (AS e HT) foi avaliado segundo diferentes índices expressos em percentual, incluindo: co-positividade $=[$ verdadeiros positivos $/$ verdadeiros positivos + falsos negativos $]$ x 100; co-negatividade $=$ [verdadeiros negativos $/$ verdadeiros negativos + falsos positivos] x 100 ; valor preditivo positivo $-\mathrm{VPP}=$ [verdadeiros positivos/total de positivos $] \times 100$; valor preditivo negativo $-\mathrm{VPN}=[$ verdadeiros negativos $/$ total de negativos] x 100; probabilidade de doença pós-parâmetro negativo $=[1-($ verdadeiros negativos/ total de negativos $)] \times 100 ; c^{-}$ positividade da análise combinada em série $=$ co-positividade do parâmetro I x co-positividade do parâmetro II e co-negatividade da análise combinada em série $=$ co-negatividade do parâmetro I + co-negatividade do parâmetro II - (co-negatividade do parâmetro I x co-negatividade do parâmetro II).

A receiver operating characteristic curve (curva ROC) foi construída aplicando-se na ordenada os valores de co-positividade e na abscissa o complemento da co-negatividade. Utilizou-se esta curva para a seleção do ponto de corte, como o valor que discrimina os resultados em positivos ou negativos. A análise da curva ROC permitiu ainda o cálculo da acurácia global dos parâmetros, avaliada através da área sob a curva ROC-ASC ${ }^{20}$. Para construir as curvas ROC para os parâmetros avaliados, utilizouse o programa estatístico MedCalc Statistical. Os intervalos de confiança de 95\% foram calculados, utilizando o programa Confidence Interval Analysis (CIA) for Windows.

O desempenho dos parâmetros fenotípicos celulares foi ainda avaliado através do índice expresso em chances denominado razão de verossimilhança $(\mathrm{RV})^{8}$, calculados através das fórmulas: $\mathrm{RV}$ para o resultado positivo $=[$ verdadeiro positivo/(verdadeiro positivo + falso-negativo)]/[falso positivo/(falso positivo + verdadeiro negativo) $]$ e $\mathrm{RV}$ para 0 resultado negativo $=[$ falsonegativo/(verdadeiro positivo + falso-negativo)]/[verdadeiro negativo/(falso positivo + verdadeiro negativo)].

\section{RESULTADOS}

Análise panorâmica do perfil dos indicadores fenotípicos celulares (\%LB, razão entre células T/B e \%CD8+HLA-DR ${ }^{+} /$ $\mathrm{CD8}^{+}$) em indivíduos soropositivos para HTLV-1. A Figura 1 mostra os valores percentuais das populações celulares do compartimento de linfócitos circulantes em indivíduos soropositivos para HTLV-1, sugeridos por Brito-Melo e cols ${ }^{1}$ como biomarcadores capazes de segregar os grupos de indivíduos assintomáticos dos portadores de HAM/TSP. A análise dos resultados evidenciou, de forma estatisticamente significativa, uma queda no \%LB, com um consequiente aumento na razão entre células T/B e 0 aumento no

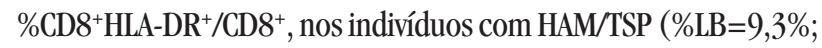
razão entre células T/B=10,4 e \%CD8 $\left.{ }^{+} \mathrm{HLA}-\mathrm{DR}^{+} / \mathrm{CD}^{+}=63,2 \%\right) \mathrm{em}$ relação ao grupo de indivíduos assintomáticos (\% $\mathrm{LB}=13,3 \%$; razão entre células T/B=6,1 e \% $\mathrm{CD} 8^{+} \mathrm{HLA}-\mathrm{DR}^{+} / \mathrm{CD}^{+}=44,3 \%$ ).

Embora esse tipo de abordagem estatística apresenta-se como importante instrumento para identificar diferenças no perfil celular entre grupos de indivíduos soropositivos para HTLV-1, na rotina de um laboratório clínico, esse tipo de abordagem possui utilização restrita uma vez que não se aplica na avaliação individual de amostras de sangue. Desta forma, torna-se necessária a utilização de métodos estatísticos apropriados para a determinação da 


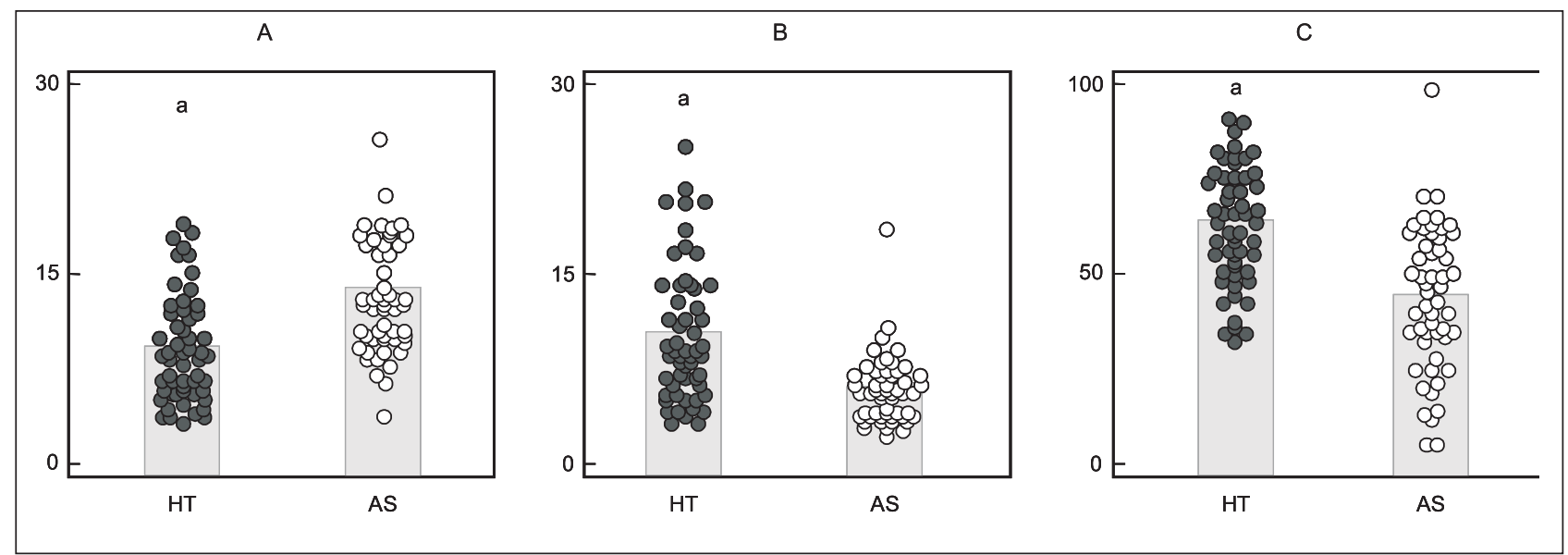

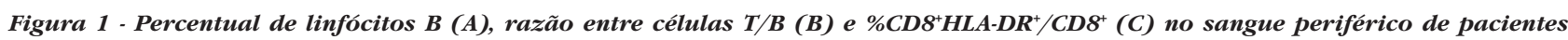
soropositivos para HTLV-1 clinicamente classificados como portadores de HAM/TSP $(H T=55)$ ou assintomáticos $($ ASO $=53)$. Os resultados estão expressos sob a forma de dispersão dos valores individuais e a média do percentual para cada grupo analisado. As diferenças estatisticamente significativas $(p<0,05)$ estão identificadas pela letra a em comparação com o grupo AS.

celularidade diferencial, em nível individual. 0 primeiro passo para essa nova abordagem, consiste na identificação do ponto de corte a ser utilizado na interpretação dos resultados do ensaio imunofenotípico.

Identificação de pontos de corte e da acurácia global para os indicadores fenotípicos celulares selecionados. Como primeiro passo para a avaliação de desempenho dos parâmetros fenotípicos \%LB, razão entre células T/B e \%CD8+HLA$\mathrm{DR}^{+} / \mathrm{CD}^{+}$através de índices de desempenho expressos em porcentagem, foi necessária a definição de um ponto de corte que permitisse classificar os resultados de cada parâmetro como positivo ou negativo. Essa definição foi realizada através da Receiver Operating Characteristic curve (curva ROC) que permite identificar, com precisão, o limiar associado a um menor número de resultados errôneos (falso-positivos e falso-negativos) ${ }^{20}$.

A Figura 2 apresenta as curvas ROC dos parâmetros fenotípicos celulares: \%LB, da razão entre células T/B e \%CD8+HLA-DR ${ }^{+}$ $\mathrm{CD}^{+}$. A localização dos melhores pontos de corte para cada parâmetro está indicada nas curvas ROC.
0 estudo da curva ROC para \%LB demonstrou que o valor de 9\% seria o melhor ponto de corte para a segregação dos grupos AS e HT. Desta forma, passa a ser considerado que valores \%LB $>9 \%$ referem-se a resultados negativos e valores de $\% \mathrm{LB} \leq 9 \%$ definem resultados positivos para esse parâmetro.

Para a razão entre células T/B, o valor de ponto de corte indicado foi 8 , considerando assim que valores da razão entre células $\mathrm{T} / \mathrm{B} \leq 8$ referem-se a resultados negativos e valores da razão entre células T/B $>8$ definem resultados positivos.

Para o parâmetro \%CD8 ${ }^{+} \mathrm{HLA}-\mathrm{DR}^{+} / \mathrm{CD}^{+}$, a curva ROC indicou como melhor ponto de corte, o valor de $50 \%$. Assim, passa-se a ser considerado que valores do $\% \mathrm{CD}^{+} \mathrm{HLA}^{-} \mathrm{DR}^{+} / \mathrm{CD}^{+} \leq 50 \%$ referem-

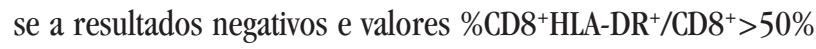
definem resultados positivos.

A curva ROC também indicou a acurácia global para os três parâmetros fenotípicos avaliados. A análise da área sob a curva demonstrou para o parâmetro \%LB um valor de $\mathrm{ASC}=0,76$ $\left(\mathrm{IC}_{95 \%}=0,67-0,84\right)$, para a razão entre células T/B, ASC $=0,77$ $\left(\mathrm{IC}_{95 \%}=0,68-0,85\right)$ e para $\% \mathrm{CD}^{+} \mathrm{HLA}^{-} \mathrm{DR}^{+} / \mathrm{CD}^{+}, \mathrm{ASC}=0,78$

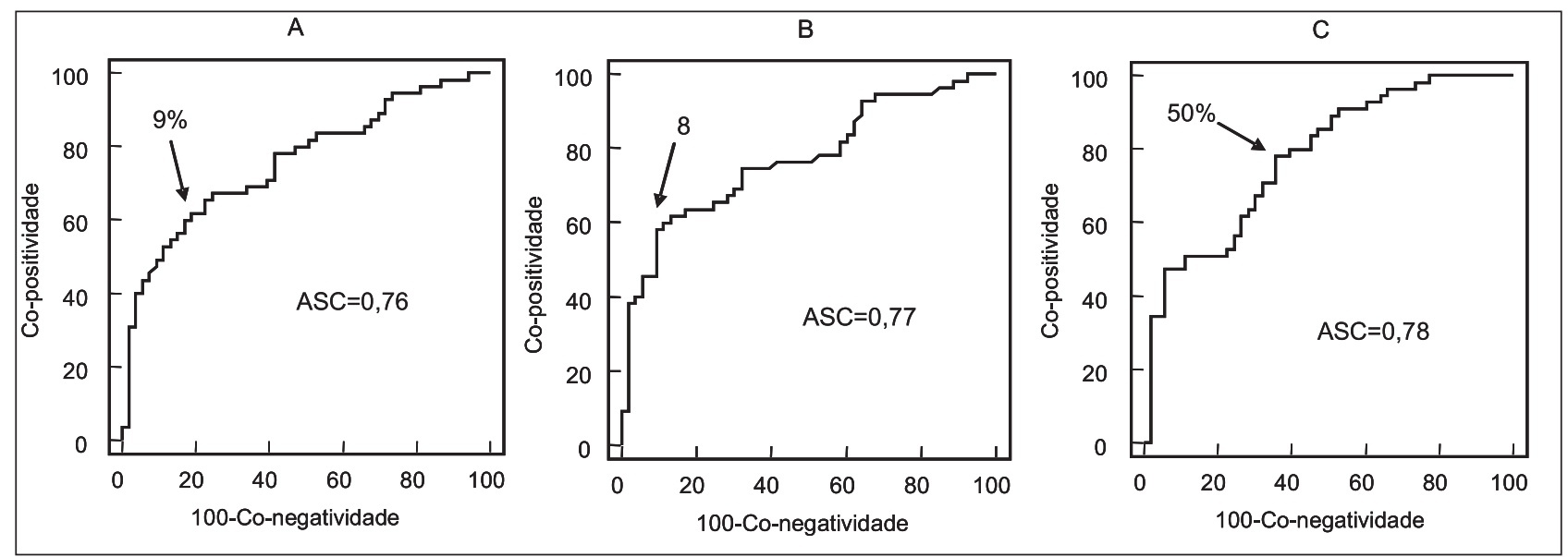

Figura 2 - Curvas ROC dos valores percentuais de linfócitos B (A), razão entre células T/B (B) e valores percentuais dos linfócitos CD8 ${ }^{+} \mathrm{HLA}_{\text {- }}$ $\mathrm{DR}^{+} / \mathrm{CDB}^{+}(\mathrm{C})$, construída a partir dos índices de desempenho, co-positividade e (100 - co-negatividade) dos parâmetros avaliados. Os resultados estão expressos pelos pontos dos gráficos que representam valores dos referidos índices avaliados, determinando assim a área sob a curva (ASC), para o percentual de linfócitos $B-A S C=0,76$, razão entre células $T / B-A S C=0,77$ e para o percentual de linfócitos $C D 8^{+} H L A-D R^{+} / C D 8^{+}-A S C=0,78$. As setas representam o melhor ponto de corte para discriminar os grupos avaliados. 
$\left(\mathrm{IC}_{95 \%}=0,69-0,86\right)$ (Tabela 1). Segundo Swets e cols ${ }^{18}$, a acurácia dos parâmetros pode ser classificada como moderada, uma vez que esta se apresenta dentro do intervalo de 0,7 a 0,9 .

Avaliação do desempenho da análise isolada dos parâmetros imunológicos selecionados para a identificação de pacientes portadores de HAM/TSP. Uma vez determinados a acurácia e o ponto de corte ideal para cada parâmetro fenotípico selecionado, foram avaliados para cada parâmetro utilizado isoladamente, os índices de desempenho: copositividade, co-negatividade, valores preditivos e probabilidade de doença (HAM/TSP - HT) pós-parâmetro negativo (Tabela 1). A análise dos dados, utilizando os pontos de corte indicados pela curva ROC, mostrou para o \%LB uma co-positividade de $60 \%$ $\left(\mathrm{IC}_{95 \%}=46-70\right)$ e uma co-negatividade de $83 \%\left(\mathrm{IC}_{95 \%}=70-92\right)$, indicando que $60 \%$ dos indivíduos com HT e $83 \%$ dos indivíduos AS apresentaram, respectivamente, \%LB inferior ou superior a 9\%. A razão entre células T/B apresentou uma co-positividade de $58 \%\left(\mathrm{IC}_{95 \%}=44-71\right)$, e uma co-negatividade de $91 \%\left(\mathrm{IC}_{95 \%}=79\right.$ 96). A análise do parâmetro \%CD8 ${ }^{+} \mathrm{HLA}-\mathrm{DR}^{+} / \mathrm{CD}^{+}$mostrou uma co-positividade de $78 \%\left(\mathrm{IC}_{95 \%}=65-88 \%\right)$ e uma co-negatividade de $64 \%\left(\mathrm{IC}_{95 \%}=46-74\right)$.

Tabela 1 - Co-positividade, co-negatividade, valores preditivos, probabilidade de doença pós-parâmetro negativo e acurácia dos parâmetros celulares isolados: \%LB, razão entre células $T / B$ e $\% C D 8^{+} H L A-D R^{+} / C{ }^{+}\left(C D 8 D R^{+}\right)$e da análise combinada da razão entre células $\mathrm{T} / \mathrm{B}$ pós \%CD8 $\mathrm{HLA}^{+} \mathrm{DR}^{+} / \mathrm{CDS}^{+}\left(\mathrm{CD8DR^{+ }}\right)$.

\begin{tabular}{lcccc}
\hline Índice & \%LB & $\begin{array}{c}\text { Razão } \\
\text { T/B(\%) }\end{array}$ & \%CD8+DR ${ }^{+}$ & $\begin{array}{c}\text { Razão T/B } \\
\text { pós \%CD8+DR }\end{array}$ \\
\hline Co-positividade & 60 & 58 & 78 & 75 \\
Co-negatividade & 83 & 91 & 64 & 74 \\
Valor preditivo positivo* & 79 & 87 & 70 & 75 \\
Valor preditivo negativo* & 67 & 68 & 74 & 73 \\
P-HTpp negativo** & 32 & 32 & 26 & 28 \\
Acurácia & 76 & 77 & 78 & 74 \\
\hline
\end{tabular}

* Valores referentes à prevalência amostral de $\mathrm{HT}=51 \%$; ** P-HTpp negativo Probabilidade de HAM/TSP pós-parâmetro negativo; \%LB - percentual de linfócitos B; razão T/B - razão entre células T/B; \%CD8 ${ }^{+} \mathrm{DR}^{+}$- percentual de células CD8 ${ }^{+} \mathrm{HLA}$ $\mathrm{DR}^{+} / \mathrm{CD} 8^{+}$.

Em conjunto, a análise dos dados demonstrou que o parâmetro

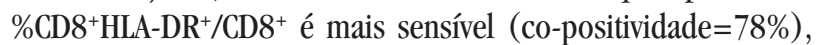
indicando sua maior capacidade em detectar os indivíduos HT. Por outro lado, a razão entre células T/B mostrou-se mais específica (co-negatividade $=91 \%$ ) em relação aos demais, tendo maior capacidade de detectar os indivíduos AS.

A análise dos valores preditivos, demonstrou que enquanto a razão entre células T/B apresentou o melhor valor preditivo positivo ( $\mathrm{VPP}=87 \%$ - $\mathrm{IC}_{95 \%}=72 \%-94 \%$; $\mathrm{VPN}=68 \%$ - $\mathrm{IC}_{95 \%}=56 \%$ 77\%), o parâmetro \%CD8 ${ }^{+} \mathrm{HLA}_{-} \mathrm{DR}^{+} / \mathrm{CD}^{+}$apresentou um maior valor preditivo negativo $\left(\mathrm{VPP}=70 \%-\mathrm{IC}_{95 \%}=57 \%-79 \%\right.$; $\left.\mathrm{VPN}=74 \%-\mathrm{IC}_{95 \%}=60 \%-84 \%\right)$. Os valores preditivos relacionados ao parâmetro \%LB não apresentaram desempenho sugestivo de sua contribuição no âmbito da monitoração clínica de indivíduos infectados pelo HTLV-1 (VPP=79\% - $\mathrm{IC}_{95 \%}=64 \%-88 \%$ e VPN=67\%$\mathrm{IC}_{95 \%}=55 \%-77 \%$ ).

A avaliação do índice "Probabilidade de doença (HAM/TSP) pós-parâmetro negativo" demonstrou que o \% $\mathrm{CD}^{+} \mathrm{HLA}^{-\mathrm{DR}^{+} / \mathrm{CD}^{+}}$ apresentou o menor valor de $26 \%$, em relação ao observado para \%LB e a razão entre células T/B (32\%).

Em suma, a análise de cada parâmetro avaliado isoladamente apresentou um desempenho moderado em discriminar indivíduos do grupo AS de pacientes do grupo HT. Nestas circunstâncias, onde cada método aplicado isoladamente apresenta valores preditivos negativos e positivos baixos e acurácia aquém do ideal, uma prática freqüente é a combinação de métodos, em paralelo (simultâneo) ou em série (seqüencial). A associação de métodos eleva a qualidade da metodologia em discriminar populações distintas, minimizando assim os resultados falso-positivos e falso-negativos.

Avaliação do desempenho da análise combinada dos parâmetros imunológicos selecionados na identificação de pacientes portadores de HAM/TSP. Para a análise combinada seqüiencial foi utilizado o parâmetro \%CD8 ${ }^{+} \mathrm{HLA}-\mathrm{DR}^{+} / \mathrm{CD}^{+}$, de elevada co-positividade, seguido pela razão entre células T/B, de maior co-negatividade. Considerando o baixo desempenho do parâmetro \%LB, este não foi incluído na análise combinada.

Para a análise combinada seqüencial, foi necessário identificar outros pontos de corte que permitissem um ganho nos índices co-positividade para \%CD8 ${ }^{+} \mathrm{HLA}-\mathrm{DR}^{+} / \mathrm{CD}^{+}$e para a razão entre células T/B em relação à análise isolada.

Desta forma, foi selecionado o ponto de corte de $30 \%$ para o

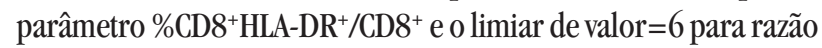
entre células $\mathrm{T} / \mathrm{B}$, com objetivo de aumentar a co-positividade destes parâmetros de $78 \%$ para $100 \%$ e de $58 \%$ para $75 \%$, respectivamente.

A escolha de novos valores limiares que favorecem a copositividade de ambos os parâmetros fundamenta-se na premissa de que um resultado falso-negativo seria mais prejudicial para o paciente, considerando a perspectiva de evolução grave da infecção pelo HTLV-1, e a necessidade de monitoração dos casos com prognóstico mais provável de progressão para HAM/TSP. Sendo assim, um indivíduo, ainda sem manifestação de sintomas de HAM/TSP, mas com alterações fenotípicas características de um paciente HT (falso-positivo), poderia ser identificado e acompanhado com uma atenção especial.

os resultados isolados de \%CD8 ${ }^{+} \mathrm{HLA}^{-\mathrm{DR}^{+} / \mathrm{CD}^{+} \text {e da razão }}$ entre células T/B, empregando os novos pontos de corte, estão apresentados na Figura 3. A análise dos dados da Figura 3A demonstrou que $100 \%$ (55/55) dos pacientes HT apresentaram

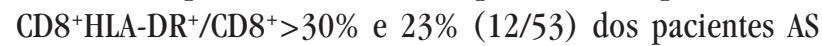

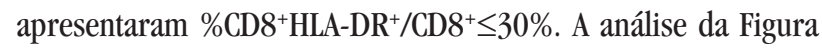
3B demonstrou que 75\% (41/55) dos pacientes HT apresentaram valores da razão entre células T/B>6 e 70\% (37/53) dos pacientes AS apresentaram valores de razão entre células $\mathrm{T} / \mathrm{B} \leq 6$.

Os resultados da análise combinada seqüiencial (razão entre

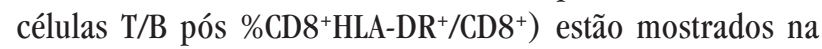
Tabela 1. Neste tipo de análise, o resultado do segundo parâmetro (razão entre células T/B) só foi avaliado quando o primeiro

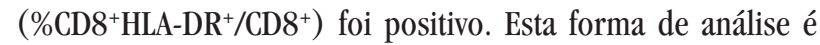
aplicada principalmente em combinações de metodologias de elevado custo, evitando-se a realização do segundo teste quando 


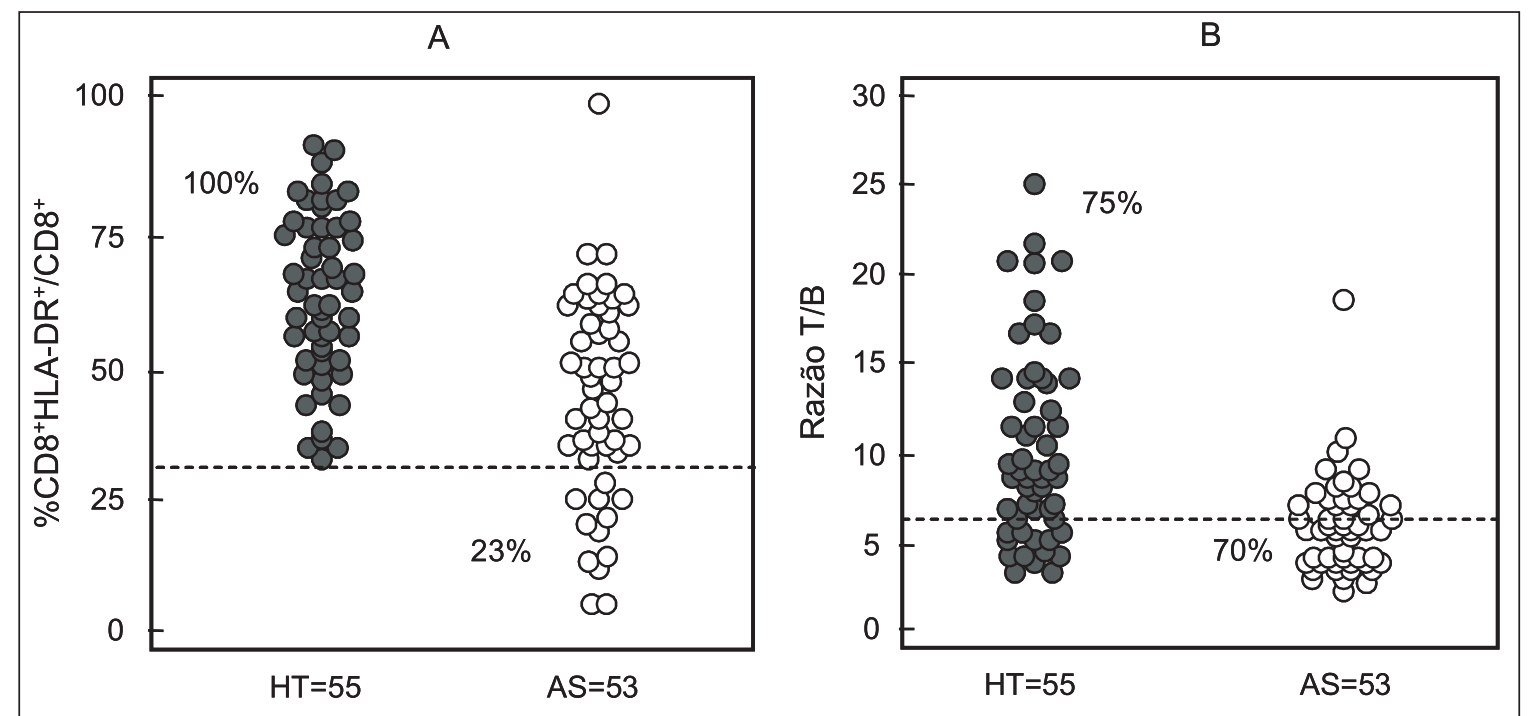

Figura 3 - Percentual de linfócitos $\mathrm{CDB}^{+} \mathrm{HLA}-\mathrm{DR}^{+} / \mathrm{CD}^{+}(3 \mathrm{~A})$ e razão entre células $\mathrm{T} / \mathrm{B}$ (3B) no sangue periférico de pacientes soropositivos para HTLV-1 clinicamente classificados como portadores de HAM/TSP (HT $=55)$ ou indivíduos assintomáticos $(A S O=53)$. Os resultados estão expressos sob a forma de dispersão dos valores individuais de cada grupo,

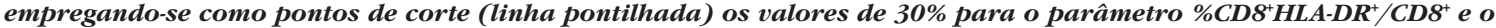
valor de 6 para a razão entre células $\mathbf{T} / B$. Resultado positivo é considerando para indivíduos acima do ponto de corte e como negativo, indivíduos abaixo do ponto de corte.

o primeiro for negativo. Assim, em nossa abordagem, o resultado final só foi considerado positivo quando os resultados de ambos os parâmetros foram positivos (maior que $30 \%$ para \%CD8 ${ }^{+} \mathrm{HLA}$ $\mathrm{DR}^{+} / \mathrm{CD}^{+}$e maior que 6 para razão entre células T/B). Resultados

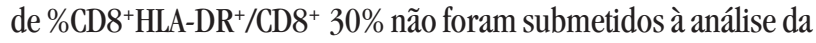
razão entre células T/B. Neste contexto, a análise combinada em série mostrou uma co-positividade de $75 \%\left(\mathrm{IC}_{95 \%}=64-82\right)$ e uma co-negatividade de $74 \%\left(\mathrm{IC}_{95 \%}=64-82\right)$ (Tabela 1). Estes dados demonstraram uma capacidade moderada da análise combinada em série em discriminar indivíduos do grupo HT dos pacientes do grupo AS. Os valores preditivos, considerando a prevalência de $51 \%$ da população em estudo, também apresentaram valores com capacidade moderada para contribuir na monitoração de HAM/TSP. A acurácia do teste foi estimada em 74\%, o que traduz a proporção de resultados corretos nos dois grupos.

Desempenho dos parâmetros imunológicos selecionados em diferentes faixas de valores, segundo as razões de verossimilhança. A razão de verossimilhança (RV) é uma abordagem que permite ampliar o espectro de informações úteis em relação àquelas fornecidas pelos índices expressos em percentagem, calculados a partir de resultado dicotômico (positivo/negativo). 0 cálculo dos valores de RVs permite a possibilidade de se trabalhar com diferentes faixas de valores de um dado parâmetro laboratorial. Considerando esta propriedade foi realizada uma análise das RVs para diferentes faixas de valores de \%LB, razão entre células T/B e \%CD8 ${ }^{+} \mathrm{HLA}_{\mathrm{L}} \mathrm{DR}^{+} / \mathrm{CD}^{+}$, com 0 objetivo de avaliar se informações adicionais poderiam ser obtidas a partir da análise individual dos parâmetros selecionados. Um ganho real no desempenho dos parâmetros propostos avaliados isoladamente foi alcançado através desse tipo de abordagem. A análise das RVs obtidas em diferentes faixas de valores revelou 0 grande valor desses indicadores imunológicos na monitoração da infecção crônica pelo HTLV-1 (Tabela 2). Os valores das RVs
Tabela 2 - Razões de verossimilhança para os valores percentuais de linfócitos $B$, razão entre células $T / B$ e percentual de células $\mathrm{CD8^{+ }} \boldsymbol{H L A}-\mathrm{DR}^{+} / \mathrm{CDB}^{+}$.

\begin{tabular}{|c|c|c|}
\hline Parâmetros fenotípicos & Pontos de corte & RVs \\
\hline & $<7 \%$ & 11 \\
\hline \multirow[t]{3}{*}{$\%$ LB } & $7<\%$ LB $\leq 13$ & 0,7 \\
\hline & $>13$ & 0,4 \\
\hline & $\leq 8$ & 0,5 \\
\hline \multirow[t]{3}{*}{ Razão entre células T/B } & $8<$ Razão entre células T/B $\leq 11$ & 2,3 \\
\hline & $>11$ & 19 \\
\hline & $\leq 30 \%$ & 0 \\
\hline \multirow[t]{2}{*}{ 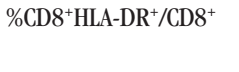 } & 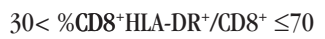 & 0,9 \\
\hline & $>70 \%$ & 10 \\
\hline
\end{tabular}

RVs - Razões de verossimilhança; \%LB - percentual de linfócitos B; \%CD8+HLA$\mathrm{DR}^{+} / \mathrm{CD}^{+}-$percentual de células $\mathrm{CD} 8^{+} \mathrm{HLA}^{-} \mathrm{DR}^{+} / \mathrm{CD}^{+}$

demonstraram que um indivíduo soropositivo para o HTLV-1 apresentando um \%LB menor que 7\%, ou razão entre células T/B

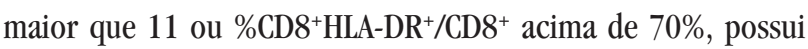
respectivamente, 11,19 e 10 vezes mais chances de pertencer ao grupo de indivíduos com HAM/TSP do que ao grupo de indivíduos AS. Por outro lado, um indivíduo soropositivo para o HTLV-1 com

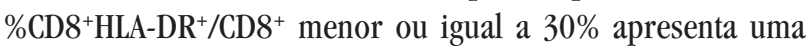
chance nula de pertencer ao grupo HT. Menor contribuição foi observada para as demais faixas de valores avaliadas.

\section{DISCUSSÃo}

Numa análise panorâmica inicial, avaliamos a dispersão dos resultados dos parâmetros fenotípicos propostos por Brito-Melo e cols ${ }^{12}$ em indivíduos infectados pelo HTLV-1, assintomáticos e portadores de HAM/TSP (Figura 1). É interessante observar que além de confirmar os resultados propostos por Brito-Melo e cols ${ }^{1}$, quando comparados aos valores de referência propostos 
na literatura para esses indicadores fenotípicos, nossos dados reforçam a hipótese de que indivíduos AS apresentam um perfil fenotípico dos parâmetros propostos $(\% \mathrm{LB}=13,3 \%$; razão entre células $\mathrm{T} / \mathrm{B}=6,1)$ semelhante à faixa de normalidade para indivíduos saudáveis com exceção da variável \%CD8 ${ }^{+} \mathrm{HLA}_{\mathrm{A}} \mathrm{DR}^{+}$/ $\mathrm{CD}^{+}$. Dados da literatura demonstram que indivíduos saudáveis apresentando de 18 a 70 anos de idade possuem \%LB=13 (variando de 11 e 16\%) e razão entre células $\mathrm{T} / \mathrm{B}=5.5$ (variando de 4.8 e 6,1$)^{5}$. Um estudo, realizado pelo nosso grupo, avaliando indivíduos residentes em Belo Horizonte demonstrou valores de $\% \mathrm{LB}=13,3 \pm 3,9 \%$ e razão entre células $\mathrm{T} / \mathrm{B}=5.8 \pm 2,41$. Os dados relativos ao \% $\mathrm{CD}^{+} \mathrm{HLA}^{\mathrm{DLR}}{ }^{+} / \mathrm{CD}^{+}$numa população de doadores de sangue soronegativos de Belo Horizonte, revelaram valores médios de $39,9 \pm 15,0 \%{ }^{2}$, inferiores ao observados para o grupo AS $(44,3 \%)$.

O desempenho dos parâmetros imunológicos celulares selecionados por Brito-Melo e cols ${ }^{2}$, foi, a princípio, avaliado empregando-se a curva ROC (Figura 2). Esse estudo demonstrou, através da área sob a curva (acurácia global), que cada parâmetro avaliado isoladamente apresenta uma capacidade moderada em discriminar indivíduos do grupo AS de pacientes do grupo HT. Segundo Swets e cols ${ }^{18}$, a acurácia global de um dado parâmetro pode ser classificada como moderada caso apresente valores entre 0,7 e 0,9 .

Numa segunda abordagem, a análise dos índices de desempenho expressos em porcentagem foi realizada, considerando os valores de pontos de corte propostos pela curva ROC (\%LB=9\%; razão entre células T/B=8 e \%CD8 ${ }^{+} \mathrm{HLA}-\mathrm{DR}^{+} / \mathrm{CD}^{+}=50 \%$ ) (Tabela 1). Nossos dados demonstraram que o parâmetro denominado razão entre células T/B mostrou-se mais específico (co-negatividade =

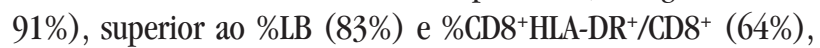
sendo mais indicado para a detecção dos indivíduos AS, sugerindo sua utilidade para confirmação da ausência de doença em estudos de monitoração. Os valores mais estáveis para a razão entre células T/B observado em indivíduos AS reforçam a hipótese de que o desequilíbrio sistêmico na resposta imune celular/humoral ocorre mais tardiamante na infecção pelo HTLV-1. 0 estudo do compartimento imune de indivíduos infectados pelo HTLV-1 tem revelado uma ampla gama de variáveis que se encontram alteradas em indivíduos portadores de HAM/TSP em relação aos assintomáticos. Embora, na história natural da infecção pelo HTLV10 fenômeno da soroconversão representa um dos eventos mais precoces, Brito-Melo e cols ${ }^{12}$ demonstraram que a redução na frequiência de linfócitos B circulantes, sugestiva da migração dessa população celular para órgãos linfóides secundários, como baço ou linfonodo, ocorre tardiamente, sendo detectada sistemicamente, no sangue periférico, apenas em indivíduos portadores de HAM/TSP. Esses resultados corroboram hipóteses prévias que demonstram a importância da monitoração de parâmetros associados à imunidade humoral como candidatos a indicadores laboratoriais aplicáveis no seguimento de pacientes infectados pelo HTLV- ${ }^{12}$. A pesquisa de outros parâmetros da imunidade humoral estão ora em andamento em nosso laboratório.

A análise do desempenho do parâmetro \%CD8+HLA$\mathrm{DR}^{+} / \mathrm{CD}^{+}$, mostrou sua característica de maior sensibilidade (co-positividade $=78 \%$ ), indicando sua grande capacidade em detectar os indivíduos HT, bem como sua habilidade de revelar alguns indivíduos AS com perfil fenotípico sugestivo de maior chance de progressão para HAM/TSP. Assim, esse parâmetro apresentou-se com grande utilidade como indicador laboratorial a ser empregado numa triagem no âmbito da monitoração de morbidade. Consistente com essa observação, nossos dados demonstraram que o parâmetro \%CD8 ${ }^{+} \mathrm{HLA}_{\mathrm{DLR}}^{+} / \mathrm{CD}^{+}$foi o único que revelou no grupo AS, valores distintos daqueles propostos na literatura como referência para indivíduos saudáveis ${ }^{15}$. Neste contexto, cabe ressaltar a importância dos eventos de ativação de linfócitos T CD8 ${ }^{+}$para os mecanismos de imunopatogênese propostos para HAM/TSP, reforçando o valor dessa variável laboratorial no contexto da monitoração de morbidade da infecção pelo HTLV-1 ${ }^{1}$.

Embora, a princípio os índices de desempenho da análise combinada (\%CD8 ${ }^{+} \mathrm{HLA}_{\mathrm{DLR}} / \mathrm{CD}^{+}$seguido da razão entre células $\mathrm{T} / \mathrm{B}$ ) tenham revelado performance moderada, numa abordagem descritiva, a análise seqüencial apresentou algumas características relevantes (Tabela 1). Foi possível confirmar que 75\% (41/55) dos pacientes HAM/TSP apresentavam valores de \%CD8 ${ }^{+} \mathrm{HLA}$ $\mathrm{DR}^{+} / \mathrm{CD}^{+}>30 \%$ e razão entre células $\mathrm{T} / \mathrm{B}>6$. Por outro lado, 74\% (39/53) dos pacientes AS ou apresentavam \%CD8+HLA$\mathrm{DR}+/ \mathrm{CD} 8+30 \%$ ou razão entre células T/B $\leq 6$.

Numa análise conjunta, o desempenho dos parâmetros imunológicos propostos apresentou uma capacidade moderada em discriminar indivíduos AS de pacientes HT, quando avaliados isoladamente ou de forma combinada seqüencial.

Numa análise complementar, o desempenho dos parâmetros propostos foi avaliado através das RVs obtidas em diferentes faixas de valores. Este novo tipo de abordagem mostrou-se de grande valor na monitoração de morbidade, apresentandose como uma perspectiva mais apropriada para a utilização dos parâmetros propostos como indicadores laboratoriais aplicáveis na propedêutica laboratorial complementar da infecção crônica pelo HTLV-1. Neste contexto, nossos dados demonstraram que um indivíduo soropositivo para o HTLV-1 com um percentual de linfócitos B menor que 7\%, uma razão entre células T/B maior que 11 e valores de \%CD8 ${ }^{+}$HLA$\mathrm{DR}^{+} / \mathrm{CD}^{+}$acima de $70 \%$, terá 11,19 e quase 10 vezes mais chances, respectivamente, de pertencer ao grupo de indivíduos com HAM/TSP (HT) do que ao grupo de indivíduos infectados (AS). Esses achados praticamente confirmam a presença de HAM/TSP, uma vez que a literatura registra que valores de RVs superiores a 10 , praticamente confirmam a presença de doença ${ }^{8}$. Por outro lado, um indivíduo soropositivo para o HTLV-1 com $\% \mathrm{CD}^{+} \mathrm{HLA}_{-\mathrm{DR}} / \mathrm{CD}^{+}$menor ou igual a $30 \%$ apresenta uma chance nula de pertencer ao grupo HT. Segundo dados da literatura, valores de RVs inferiores a 0,1 são praticamente confirmatórios para a ausência de doença ${ }^{8}$. Estudos prévios em nosso laboratório, empregando esse tipo de abordagem já haviam sugerido que a categorização dos valores dos parâmetros fenotípícos selecionados em diferentes faixas de valores seria uma abordagem promissora para a segregação dos indivíduos soropositivos para o HTLV-1 em AS e HT' ${ }^{1}$. 
Estes dados sugerem que o monitoramento desses fenótipos celulares por meio da técnica de citometria de fluxo, empregando a estratégia de diferentes faixas de valores poderia ser um importante instrumento laboratorial auxiliar no prognóstico clínico da doença neurológica degenerativa associada à infecção pelo HTLV-1. No entanto, é importante enfatizar que nossa proposta não sugere a utilização desses parâmetros imunológicos como marcadores definitivos de HAM/TSP, mas como parâmetros laboratoriais alternativos e adicionais capazes de contribuir para a monitoração clínica da desordem neurológica associada com a infecção por HTLV-1, de maneira semelhante à utilização do parâmetro fenotípico $\% \mathrm{CD}^{+}{ }^{+}$no sangue periférico como indicador laboratorial do perfil de imunidade celular sugerido para monitoração de pacientes infectados pelo HIV. Neste contexto, o parâmetro proposto \%CD4+ não deve ser utilizado isoladamente como marcador conclusivo de um status clínico considerando que outras condições patológicas humanas podem também promover alterações no perfil fenotípico de leucócitos circulantes.

No âmbito da infecção pelo HTLV-1, variáveis como o tempo de evolução da infecção, co-infecções e a presença de situações clínicas concomitantes (manifestações dermatológicas, oftalmológicas e psicológicas associadas à infecção pelo HTLV-1) inerentes à forma assintomática ou mesmo os diferentes graus de morbidade observados entre pacientes portadores de HAM/TSP poderiam ser variáveis de confusão nesse tipo de análise. Desta forma, devem-se estimular estudos futuros de validação do uso dos parâmetros fenotípicos propostos através de estudo de seguimento longitudinal de diferentes coortes. Um estudo piloto de caráter longitudinal encontra-se ora em andamento em nosso laboratório. Neste espectro amplo de manifestações clínicas da infecção crônica pelo HTLV-1 chamamos a atenção para a necessidade de segregação dos indivíduos AS e dos pacientes HT em subgrupos clínicos para a confirmação do desempenho dos parâmetros celulares avaliados, lembrando que a interpretação de dados derivados de imunofenotipagem deve ser realizada com cautela, uma vez que esses apresentam de natureza inespecífica. Assim, a inclusão seletiva e subcategorizada de indivíduos com sorologia positiva para o HTLV-1 numa abordagem fenotípica celular complementar, minimizaria a interferência que outros agentes infecciosos ou condições patológicas podem exercer sobre os valores relativos e absolutos dessas subpopulações celulares.

\section{REFERÊNCIAS}

1. Brito-Melo GE, Martins-Filho OA, Carneiro-Proietti AB, Catalan-Soares B, Ribas JG, Thorum GW, Barbosa-Stancioli EF. Phenotypic study of peripheral blood leucocytes in HTLV-1-infected individuals from Minas Gerais, Brazil. Scandnavian Journal of Immunology 55:621-628, 2002.

2. Brito-Melo GE, Souza JG, Barbosa-Stancioli EF, Carneiro-Proietti AB, CatalanSoares B, Ribas JG, Thorum GW, Rocha RD, Martins-Filho OA, Grupo Interdisciplinar de Pesquisas em HTLV. Establishing phenotypic features associated with morbidity in human T-cell lymphotropic virus type 1 infection. Clinical Diagnostic and Laboratory Immunology 11:1105-1110, 2004.

3. Carneiro-Proietti ABF, Ribas JGR, Catalan-Soares BC, Martins ML, Brito-Melo GEA, Martins-Filho OA, Pinheiro SR, Araújo AQC, Galvão-Castro B, Oliveira MST, Guedes AC, Proietti FA. Infecção e doença pelos vírus linfotrópicos humanos de células T (HTLV-1/2) no Brasil. Revista da Sociedade Brasileira de Medicina Tropical 35: 499-508, 2002.

4. Catalan-Soares B, Carneiro-Proietti AB, Proietti FA, Interdisciplinary HTLV Research Group. Heterogeneous geographic distribution of human T-cell lymphotropic viruses I and II (HTLV-I/II): serological screening prevalence rates in blood donors from large urban areas in Brazil. Cadernos de Saúde Pública 21:926-931, 2005.

5. Hannet I, Erkeller-Yuksel F, Lydyard P, Deneys V, DeBruyere M. Developmental and maturational changes in human blood lymphocyte subpopulations. Immunology Today 13:215-218, 1992.

6. Jacobson S, Krichavsky M, Flerlage N, Levin M. Immunopathogenesis of HTLV-1 associated neurologic disease: massive latent HTLV-1 infection in bone marrow of HAM/TSP patients. Leukemia 3 (suppl): 73-75, 1997.

7. Jacobson S, Shida H, Mcfarlin E, Fauci AS, Koenig S. Circulating CD8 + cytotoxic T lymphocytes specific for HTLV-1 $\mathrm{pX}$ in patients with HTLV-1 associated neurological disease. Nature 348: 245-248, 1990.

8. Jaeschke R, Guyatt GH, Sackett DL. Users' guides to the medical literature. III. How to use an article about a diagnostic test. B. What are the results and will they help me in caring for my patients? The Evidence-Based Medicine Working Group. The Journal of the American Medical Association 271:703-707, 1994.

9. Kajiyama W, Kashiwagi S, Ikematsu H, Hayashi J, Noruma H, Okochi K. Intrafamilial transmission of adult T-cell leukemia virus. Journal of Infectious Diseases 154:851-857, 1986

10. Kitajima I, Yamamoto K, Sato K, Nakajima Y, Nakajima T, Maruyama I, Osame M, Nishioka K. Detection of human T cell lymphotropic virus type I proviral DNA and its gene expression in synovial cells in chronic inflammatory arthropathy. Journal of Clinical Investigation 88:1315-1322, 1991.

11. Kubota R, Soldan SS, Martin R, Jacobson S. Selected cytotoxic T lymphocytes with high specificity for HTLV-1 in cerebrospinal fluid from a HAM/TSP patient. Journal of Neurovirology 8:53-57, 2002.

12. Macatonia SE, Cruickshank JK, Rudge P, Knight SC. Dendritic cells from patients with tropical spastic paraparesis are infected with HTLV-1 and stimulate autologous lymphocyte proliferation. AIDS Research and Human Retroviruses 8:1699-1706, 1992.

13. Murphy EL, Figueroa P, Gibbs WN, Brathwaite A, Holding-Cobham M, Waters D, Cranston B, Hanchard B, Blattner WA. Sexual transmission of human Tlymphotropic virus type-I. Annals of Internal Medicine 111:555-560, 1989.

14. Osame M, Usuku K, Izumo S, Ijichi N, Amitani H, Igata A, Matsumoto M, Tara M. HTLV-1 associated myelopathy, a new clinical entity. Lancet 1: 1031-1032, 1986.

15. Poiesz BJ, Ruscetti FW, Reitz MS, Kalyanaraman VS, Gallo RC. Isolation of a new type C retrovirus (HTLV) in primary uncultures cells of a patient with Serary T cell leukemia. Nature 294:268-271, 1981.

16. Popovic M, Reitz MS, Sarngadharan MG. The virus of Japanese adult T-cell leukaemia is a member of the T-cell leukaemia virus group. Nature 300:63-66, 1982.

17. Proietti FA, Carneiro-Proietti AB, Catalan-Soares BC, Murphy EL. Global epidemiology of HTLV-1 infection and associated diseases. Oncogene 24:6058$6068,2005$.

18. Swets JA. Measuring the accuracy of diagnostic systems. Science 240:1285-1293, 1988

19. Taylor GP. Pathogenesis and treatment of HTLV- 1 associated myelopathy. Sexually Transmitted Infections 74:316-322, 1998.

20. Zweig MH, Campbell G. Receiver-operating characteristic (ROC) plots: a fundamental evaluation tool in clinical medicine. Clinical Chemistry 39:561-577, 1993. 